1 D. H. LEHMER, $M T A C$, p. 133-135.

${ }^{2}$ G. N. Watson, $A$ Treatise on the Theory of Bessel Functions, Cambridge, 1922, p. 228.

\title{
RECENT MATHEMATICAL TABLES
}

158[A].-H. S. UhLer, Exact Values of the first 200 Factorials, New Haven, Conn., privately published by H. S. Uhler, 1944,24 p. + cover. $16 \times 25.5$ cm. Photo-litho print. $\$ .80$

In $M T A C$, p. 163,125 , are records of unpublished tables of $n$ ! by JoFFE, $n \leqslant 100$, and by Salzer and Hillman, $n \leqslant 120$. Among previously printed tables the most extensive are those of Peters and Stein (J. T. Peters, Zehnstellige Logarithmentafel . . ., v. 1, 1922, Anhang, p. 58), $n \leqslant 60$; of F. RobBins, R. So. Edinburgh, Trans., v. 52, 1917, p. 167-174, $n \leqslant 50$; and of L. Potin, Formules et Tables Numériques . . ., Paris, 1925, p. 836, $n \leqslant 50$ (with four errors). The Introduction of the table under review, $n \leqslant 200$, (p. [iii-vi]) includes an account of "details of computation," "additional checks," "comparison with the results of others," "peculiarities of the table."

159[A].-Werner F. Vogel, Angular Spacing Tables, Detroit, Michigan, Vinco Corporation, 1943. iv, 233 p., hinged. $21.8 \times 28.5 \mathrm{~cm} . \$ 10.00$

Especially in connection with the spacing of teeth in such things as precision gears, splines, and index plates, are modern demands of a very high order. It is desirable to know within $0^{\prime \prime} .0005$ how many degrees, minutes and seconds are contained in angles between a line from the center of the gear through the center of one tooth to the line from the center of the gear through the center of any subsequent tooth on the gear. The main table of this volume (p. 1-208) provides such information for gears with 2(1)200 teeth. The presentation of the material is very clear. While seconds are given to $3 \mathrm{D}$, in every case where this decimal part is $\geqslant .5$ the entry appears as, for example, $35 .^{*} 510$, and the following footnote appears on each page: "*Star indicates that one second must be added when decimals are disregarded." For all practical purposes accuracy to the nearest 1 " suffices.

In the next three tables (p. 212-217), the values are given for $90^{\circ} / n, 180^{\circ} / n, 360^{\circ} / n$ where $n$ is the number of sides of a regular polygon, $n=4(1) 200$. In the first table the values of these angles are in degrees, minutes, and seconds to $4 \mathrm{D}$; in the second table they are given in degrees to $7 \mathrm{D}$; and in the third table in radians to $10 \mathrm{D}$. In each of these tables values corresponding to $n=1(1) 3$ are also given. On p. 218-227 is an 8-place table for converting any number of minutes and seconds up to $1^{\circ}$ into a decimal of a degree. The next table is for the conversion of $0\left(0^{\prime \prime} .001\right) 0^{\prime \prime} .999$ to decimals of a degree, $8 \mathrm{D}$. Under the heading "Important Constants," $\pi$ and $1 / \pi$ are given to $70 \mathrm{D} ; n \cdot \pi$ and $n \cdot(1 / \pi), n=1(1) 9$, to $35 \mathrm{D}$; $\pi / n$ and $1 /(n \pi), n=1(1) 19$, to $35 \mathrm{D}$; and $n \cdot \pi / 180$ and $n \cdot 180 / \pi, n=1(1) 9$, to 35D.

Mr. Vogel is now a member of the staff of Wayne University Engineering College in Detroit. As a former member of the Computing staff of J. T. Peters at the Astronomisches Recheninstitut of Berlin-Lichterfelde, we may be sure that he was most meticulous in every type of check to ensure the accuracy of his published tables.

R. C. A.

160[D, P].-General Electric Co., Trigonometric Functions of Half Center Angle of Regular Polygons (Standard Tables Division, Design Data, Mathematical Tables, Section G 902.4), February 22, 1944. 10 p. $20.5 \times 26.5 \mathrm{~cm}$. 
If $n$ equals the number of sides of a regular polygon $180^{\circ} / n$ is the number of degrees in half the center angle, subtended by a side. For $n=3(1) 500$ the angles $180^{\circ} / n$ are expressed to the nearest $0^{\prime \prime} .01$. Then follow 7-place values of their natural sines, cosines, tangents and secants. Such tables are used in manufacture of machines with integral numbers of commutator bars or slots. An earlier edition of this table (1943) was of $m$ sin $\left(180^{\circ} / n\right)$, only for $n=3(1) 500, m=1,2,3$.

$$
\text { R. C. A. }
$$

161[F].-J. A. Todd, "A table of partitions," London Math. So., Proc., v. 48,1943 , p. $229-240 ; 1944$, p. $241-242$.

The author denotes by $p(n, m)$ the number of partitions of $n$ into precisely $m$ parts, and gives a table of $p(n, m)$ for $m \leqslant(n+1) / 2$ and for $n \leqslant 100$. This is an extension of an old table of EULER ${ }^{1}$ which gives the same function for $m \leqslant 20$ and $n \leqslant 59$.

It is easily seen that $p(n, m)$ is also the number of partitions of $n$ in which the largest part is $m$. By considering separately the cases in which $m$ occurs once and more than once as the largest part, we see at once that

$$
p(n, m)=p(n-1, m-1)+p(n-m, m) .
$$

This is the recurrence relation by means of which the author has calculated his table. It is clear that if $m>n / 2$ the partitions enumerated by $p(n, m)$ will involve $m$ only once, the remaining parts giving an unrestricted partition of $n-m$; that is

$$
p(n, m)=p(n-m), \quad m>n / 2,
$$

where $p(k)$ is the number of unrestricted partitions of $k$. This fact enables the author to dispense with one half of the values of $p(n, m)$ by giving a small auxiliary table of $p(k)$ for $k \leqslant 50$.

The reviewer has compared the table with that of Euler and has found complete agreement where they overlap.

Interest in the present table is heightened by quite recent investigations of GUPTA, ${ }^{2}$ ERDös \& LEHNER ${ }^{3}$ and others on the function $p(n, m)$. The author, who is apparently unaware of these results, states that $p(n, m)$ is of the "order of magnitude" $n^{m-1} /[m !(m-1) !]$ for fixed $m$ as $n \rightarrow \infty$. Gupta has shown much more, namely

$$
\frac{1}{m !}\left(\begin{array}{c}
n-1 \\
m-1
\end{array}\right) \leqslant p(n, m) \leqslant \frac{1}{m !}\left(\begin{array}{c}
n-1+c \\
m-1
\end{array}\right)
$$

where $c$ can be taken as small as $m(m-1) / 4$, and perhaps slightly less. Another important question of current interest on which this table may shed some light is that of the maximum value of $p(n, m)$ as a function of $m$. In other words, among all partitions of $n$ what size for the largest part is most popular, or to put it another way, what is the most popular number of parts? For $n=90$ the table shows a maximum at $m=17$. In fact $p(90,17)=3483945$. However a very close second is $p(90,16)=3483604$. According to the theory the answer is approximately

$$
(3 n / 2)^{3} \cdot[\log (n / \pi)]
$$

which for $n=90$ gives 16.64 .

A companion double-entry table of restricted partitions is that of GuPTA ${ }^{4}$ which gives for $n \leqslant 300$ the number $q(n, m)$ of partitions of $n$ with $m$ as the smallest element or, what is the same, the number of partitions of $n$ in which the largest part occurs precisely $m$ times. This perhaps less natural function satisfies the recurrence formula

$$
q(n, m)=q(n-m, m)+q(n+1, m+1)
$$


and was used by GuPTA ${ }^{4}$ (proceeding by descent on $m$ ) to construct a table of $p(n)=q(n+1,1)$ for $n \leqslant 600$.

D. H. L.

${ }^{1}$ L. Euler, "De partitione numerorum," Acad. Sci. Petrop., Novi Commentarii, v. 3 (1750-1), 1753, p. 125-169. Commentationes Arithmeticae. St. Petersburg, v. 1, 1849, p. $73-$ 101, Opera Omnia, s. 1, v. 2, 1915, p. 254-294. Chrystal in his algebra refers to a smaller table of Euler in his Introductio in Analysin Infinitorum [Lausanne, 1748, chapter 16; Opera Omnia, s. 1, v. 8, 1922, p. 327-328] and many later writers, including the author, have followed this reference.

${ }^{2}$ H. GuPTA, "An inequality in partitions," Bombay Univ., Jour., v. 9, (1942), p. 16-18.

3 P. ERDös \& J. LEHNER, "The distribution of the number of summands in the partitions of a positive integer," Duke Math. J., v. 8, 1941, p. 335-345.

${ }^{4} \mathrm{H}$. Gupta, Tables of Partitions, Madras, 1939.

162[I].-NYMTP, A. N. LowAN, technical director, Tables of Lagrangian

Interpolation Coefficients, New York, Columbia University Press, 1944, xxxvi, 392 p., $19.6 \times 26.5 \mathrm{~cm}$. Reproduced by a photo offset process. $\$ 5.00$.

This interesting volume gives tables of the coefficients $A_{k}^{(n)}(p)$ of Lagrange's interpolation formula for equally spaced arguments:

$$
f(a+p h)=\sum_{k=1-\nu}^{n-\nu} A_{k}^{(n)}(p) f(a+k h)+R_{n}(f)
$$

where $\nu=[(n+1) / 2]$, and $h$ is the interval of the argument for which $f$ is tabulated. The actual formula for $A_{k}^{(n)}(p)$ can be written

$$
A_{k}^{(n)}(p)=\frac{(-1)^{n+k+\nu} \Gamma(p+\nu)}{(p-k) \Gamma(p-n+\nu) \Gamma(n-\nu-k+1) \Gamma(\nu+k)}
$$

from which it is clear that $A_{k}^{(n)}(p)$ is a polynomial in $p$ of degree $n-1$. The values of these coefficients are tabulated for

$$
\begin{gathered}
1-\nu \leqslant k \leqslant n-\nu \\
3 \leqslant n \leqslant 11
\end{gathered}
$$

The range of the argument $p$ varies with $n$ according to the following scheme:

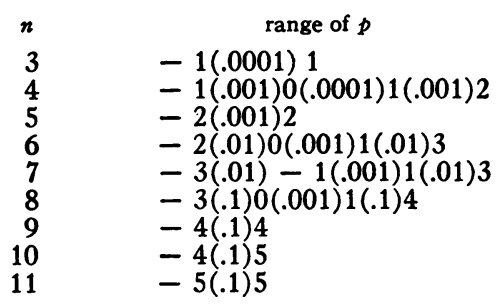

From this it is seen that of these nine tables the first two are the main ones and that for $n<9$ the important range, between 0 and 1 , is pretty finely divided.

There are two other tables of $A_{k}^{(n)}(p)$ intended for use in subtabulation. These are for $n \leqslant 9$ and give exact values of $A_{k}^{(n)}(p)$ for $p$ at intervals of .1 and $1 / 12$. With these tables a given numerical table may be enlarged $m$-fold by a single subtabulation for $m=2,3,4,5$, $6,10,12$.

Throughout the volume all values which are not exact are given to 10 decimal places.

Finally there are two other tables for use in approximate integration. These give the polynomials

$$
M_{k}^{(n)}(p)=\int_{0}^{p} A_{k}^{(n)}(t) d t
$$


and the coefficients

$$
M_{k}^{(n)}(m+1)-M_{k}^{(n)}(m)
$$

for integers $m, k, n$ satisfying

$$
1-\nu \leqslant m \leqslant n-\nu-1, \quad 1-\nu \leqslant k \leqslant n-\nu, \quad 3 \leqslant n \leqslant 11 .
$$

There is a bibliography of 8 items describing the principal previous tables of Lagrangean coefficients. There is no mention of the obscure table of LiTTLE nor of the two places where parts of the volume under review were published earlier; see $M T A C, \mathrm{p} .185$.

The introduction is quite interesting. It contains a brief discussion of the properties of the $A$ 's and their relations to coefficients of other interpolation formulae based on differences. There is an adequate treatment of the upper bound for the remainder $R_{n}(f)$ and other errors in interpolation. Finally quadrature formulae based on the Lagrangean interpolation scheme are derived and discussed.

The reviewer has recently set forth reasons $(M T A C$, p. 184) why he believes that interpolation without differences by the Lagrangean method will become more popular with users of good computing machines. This splendid volume will do much to enhance such popularity. It is a "must" for the library of every mathematical laboratory.

D. H. L.

163[I].-H. E. SALZER, "Table of coefficients for inverse interpolation with advancing differences," J. Math. Phys., M.I.T., v. 23, 1944, p. 75-102. $17.5 \times 25.5 \mathrm{~cm}$.

This table of coefficients is a companion to a previous one ${ }^{1}$ by the author based on the more popular central difference set up. The present table will be found more fundamental in its application since advancing differences require a minimum amount of knowledge of the given function. One associates inverse interpolation with the discovery of a real root of some equation $f(x)=$ const. Often $f(x)$ is not a function which has been tabulated and published. This means that a "home-made" table of just a few entries is needed. Thus one is of ten working at the beginning of a table.

Let $y=f(x)$ be a monotone function of $x$ tabulated for $x=x_{0}, x_{0}+h, x_{0}+2 h, \cdots$. If $\boldsymbol{y}$ is a given value, the problem of inverse interpolation is that finding a number $p(0<p<1)$ such that $f\left(x_{0}+p h\right)=y$. Let $D_{k}=\frac{\Delta^{k} f\left(x_{0}\right)}{\Delta f\left(x_{0}\right)}$ and $m=\frac{f\left(x_{0}+p h\right)-f\left(x_{0}\right)}{\Delta f\left(x_{0}\right)}$ so that $0<m<1$; then the Gregory-Newton formula gives

$$
p=m-\sum_{\nu=2}\left(\begin{array}{l}
p \\
\nu
\end{array}\right) D_{\nu}
$$

Complete reversion of this series for $p$ gives

$$
\begin{aligned}
p=m & -\left(\begin{array}{c}
m \\
2
\end{array}\right) D_{2}-\left(\begin{array}{l}
m \\
3
\end{array}\right) D_{3}+\frac{m\left(m-\frac{1}{2}\right)(m-1)}{2} D_{2}^{2}-\left(\begin{array}{l}
m \\
4
\end{array}\right) D_{4} \\
& +\cdots+P\left(m \mid r_{1}, r_{2} \cdots\right) D_{r_{1}}^{\alpha_{1}} D_{r_{2}}^{\alpha_{2}} \cdots+\cdots .
\end{aligned}
$$

Here the general term is said to be of order $r_{1} \alpha_{1}+r_{2} \alpha_{2}+\cdots$ and the coefficient $P(m)$ is a polynomial in $m$. The first two pages of this paper give the explicit expansion through the terms of the 8 th order, only 22 terms in all. This is a slight extension of a previous result of H.T.D. ${ }^{2}$ now confirmed except for two minor errata. ${ }^{2}$ The purpose of the present paper is to give a table of these 22 coefficients $P(m)$. Actually the table begins with the fourth order terms since the earlier terms are easy to calculate and have been tabulated of ten. (See bibliography of Lagrangean interpolation coefficients $M T A C$, p. 184-5, also the previous review, RMT 162). The remaining 19 coefficients are naturally arranged according to their order. They are given to ten decimals for $0 \leqslant m \leqslant 1$ at intervals of .001 for the 4th and 5th order terms, .01 for 6 th order terms and .1 for the 7 th and 8 th order terms. 
It may be worth noting that the reversion of (1) accomplished once for all for a general $m$ in (2) has in the past been done numerically for a fixed $m$ by the method of iteration. This table should be of considerable help in handling problems in which a large number of approximate roots for some complicated transcendental equations are needed, as for instance in the determination of the characteristic values of some boundary value problem.

D. H. L.

1 "Table of coefficients for inverse interpolation with central differences," J. Math. Phys., v. 22, 1943, p. 210-224.

2 H. T. DAvis, Tables of the Higher Mathematical Functions, v. 1, Bloomington, Ind., 1933, p. 80-81. Mr. Salzer notes "The formula obtained by Davis was checked and found to be entirely correct except for two obvious printing errors, namely, p. 81 line 6 for $3 !^{2}$ read $4 !^{2}$ and on line 7 for +36 read $-36 . "$

164[I, K].-R. A. Fisher \& F. YATES, Statistical Tables for Biological, Agricultural and Medical Research, Edinburgh, Oliver and Boyd, I. first ed., 1938, viii, 91 p.; II. second ed., revised and enlarged, 1943, viii, 98 p. $21.9 \times 28.1 \mathrm{~cm}$. $13 \mathrm{~s}$. $6 \mathrm{~d}$.

This volume is a collection of those tables which, in the experience of the authors, have been found most frequently useful in the application of statistical methods to biology, agriculture and medicine. Since they cover a wide range of applications, the tables will be reviewed according to their subject matter, rather than in the order in which they appear in the book.

Most of the tables refer to uses either of the analysis of variance or of the theory of statistical regression. The first seven tables are reprinted, with exceptions noted below, from R. A. Fisher, Statistical Methods for Research Workers, Edinburgh, Oliver and Boyd, first ed. 1925; eighth ed. 1941.

Table. I. The argument $P$ is the probability that a normal deviate, with zero mean and unit standard deviation, falls outside the limits $(-x,+x)$. For $P=0(.01) .99$, $x$ to $6 \mathrm{D}$; for $P=.0^{2} 1, .0^{3} 1$, to $.0^{8} 1, x$ to $5 D$.

Table II. Contains the ordinates of the standard normal distribution to $4 \mathrm{D}$, for the deviate $x=0(.01) 3(.1) 3.9$. Tables III to VII were developed originally for the common tests of significance associated with the analysis of variance, namely the $t, \chi^{2}$, and $z$ tests and the test of a product-moment correlation coefficient $r$.

Table III. For "Student's" $t$ distribution with $n$ degrees of freedom, RMT 111, $t$ is given (to 3D) such that the probability is $P$ of a random variate falling outside the limits $(-t,+t)$. $P=.9(-.1) .1, .05, .02, .01, .001 ; n=1(1) 30,40,60,120, \infty$. A table of $t$ to 5D is described in RMT 111.

For a description of Table IV, significance levels of the $\chi^{2}$ distribution, see RMT 101, and for Table V, significance levels of the variance-ratio and $z$ distributions, see RMT 99. The variance-ratio tables and the table of $z$ for $P=.2$ do not appear in Fisher, loc. cit.

Table VI. In a bivariate normal distribution where the population correlation coefficient $\rho$ is zero, the distribution of the sample product-moment correlation coefficient $r$ is related to that of "Student's" $t$ by the equation $t=r \sqrt{n} / \sqrt{1-r^{2}}$ where $t$ has $n$ degrees of freedom and $(n+2)$ is the number of pairs of values from which $r$ is calculated. In Table VI, $P$ is the probability that a sample correlation coefficient falls outside the limits $(-r,+r) . P=.1, .05, .02, .01, .001 ; n=1(1) 20(5) 50(10) 100 ; r$ to $4 \mathrm{D}$ throughout most of the table.

Table VII. Contains $r=\tanh z$, for $z=0(.01) 3(.1) 4.9 ; r$ to $4 \mathrm{D}$ for $z$ less than 1.8 , to $5 \mathrm{D}$ thereafter. This transformation is useful for tests of significance involving $r$ in cases where $\rho$ is not zero; for example, a test of the hypothesis that two populations have the same correlation coefficient. When $\rho$ is near \pm 1 , the distribution of $r$ is markedly skew even in moderately large samples; $z$, on the other hand, is approximately normally distributed with mean $\tanh ^{-1} \rho$ and variance $1 /(n-1)$ where $n$ is as before two less than the number of pairs. 
The techniques and tests associated with the analysis of variance are based on the assumption that the data follow the normal distribution. In the development of statistical techniques for problems where this assumption is far from true, one approach is to transform the data to a scale in which analysis of variance techniques can be applied, at least as a first approximation to which further refinements may be added if required. For the research worker who is not an expert statistician this device has obvious advantages, since a few techniques can be used repeatedly. We consider now three groups of tables which facilitate applications of this type.

In certain toxicological experiments it is considered that the number of subjects which succumb to a given dose (but survive any lesser dose) follows a normal distribution when plotted against a suitable function of the dose, usually the log. Thus in a group of subjects the fraction $P$ which is killed by a dose is given theoretically by the relation

$$
P=\frac{1}{\sigma \sqrt{2 \pi}} \int_{-\infty}^{d} e^{-(t-m)^{2} / 2 \sigma^{2}} d t=\frac{1}{\sqrt{2 \pi}} \int_{-\infty}^{x} e^{-\frac{1}{2} t^{2}} d t .
$$

where $x=(d-m) / \sigma$ and $d$ is the log dose. Where this theory holds, the observed fraction $P$ in any experiment, when transformed to the normal deviate $x$, shows a linear relation with $\log$ dose, the slope of the line providing an estimate of $1 / \sigma$. From estimates of $m$ and $\sigma$ the dose required to kill any desired fraction and the relative performance of two poisons can be predicted. In Table IX, $P$ (in percents) $=0.1(0.1) 98.0(0.01) 99.99$; the normal deviate $x$ is given to $4 \mathrm{D}$ with the addition of a constant value 5 in order to avoid negative deviations. The table is reprinted from C. I. Bliss, Ann. Appl. Biol., v. 22, 1935, p. 138140, with the inclusion of first differences. Apart from the constant 5 and from the fact that $P$ refers to only a single tail, this table is simply a more extensive form of Table $I$. Table $\mathrm{X}$, for the same purpose, gives $(-x)$ to $4 \mathrm{D}$ for all proper fractions $P\left(\leqslant \frac{1}{2}\right)$ with denominators up to 30 . There appears to be an error in the footnote to Table IX; the words "exceeded by" should be replaced by "which exceeds."

Table XI applies to later stages in the calculations. For $Y=(x+5)=5.0(0.1) 8.9$, the functions $Y+Q / Z(4 \mathrm{D}), 1 / Z$ (mostly $5 \mathrm{~S}$ ) and $Z^{2} / P Q(5 \mathrm{D})$ are given; $Q=1-P$ and $Z$ is the ordinate of the normal curve at the point $\mathrm{P}$. The function $Z^{2} / P Q$ supplies the weights for fitting the line while the other two functions appear in further refinements.

Table XII gives $\sin ^{-1} \sqrt{p}=\varphi$ expressed in degrees to $1 \mathrm{D}$, for $p$ (in percents) $=0$ (1)99 and Table XIII gives the same function for all proper fractions $\left(\leqslant \frac{1}{2}\right)$ with denominators up to 30. If it is desired to apply the analysis of variance to data expressed as fractions or percentages, where the errors follow the binomial distribution, the angular transformation provides a scale in which the error variances are more nearly equal at all points. The analysis of variance in angles may be regarded as a first approximation to the maximum likelihood analysis and is often adequate. For more precise work the maximum likelihood solution itself may be obtained by means of adjustments to the angles given in Table XIV. The relevant functions are $\varphi+(90 / \pi) \cot \varphi$ and $(180 / \pi) \csc 2 \varphi$, each given to $1 \mathrm{D}$ for $\varphi=45^{\circ}\left(1^{\circ}\right) 89^{\circ}$.

Table XX supplies a transformation for the analysis of variance of ranked data, that is, data in which only the order $1,2,3, \cdots$ of preference or performance is recorded. The table shows to $2 \mathrm{D}$ the mean value $n_{r}$ of the $r^{\text {th }}$ largest of a sample of $n$ from a normal distribution with mean zero and unit standard deviation: $n=2(1) 50 ; r \leqslant \frac{1}{2} n$. Values for $r>\frac{1}{2} n$ are obtained by symmetry. The function involved is

$$
n_{r}=\int_{0}^{1} \frac{n !}{(r-1) !(n-r) !} p^{n-r} q^{r-1} x d p
$$

where $p$ is the probability that a normal deviate is less than $x$. The sums of squares of the mean deviations are given for $n=2(1) 50$ in Table XXI. The assumption involved in the use of Table $\mathrm{XX}$ is that if the ranked scores could be replaced by exact numerical scores, the latter would follow a normal distribution. When this assumption is valid, estimates made in the transformed scale are more efficient than those made in the original scale. 
Many methods have been developed for fitting a polynomial regression in $x$ when $x$ takes the values $1,2, \cdots, n$. Table XXIII, which contains the orthogonal polynomials $\xi_{r}^{\prime}(x)$, for $n=3(1) 52, r=1(1) 5$, is the basis of a procedure that is convenient and expeditious when a multiplying machine is available. Compare RMT 129. If $\xi_{0}(x)=1$, $\xi_{1}(x)=x-x, \xi_{r+1}(x)=\xi_{1}(x) \xi_{r}(x)-\frac{r^{2}\left(n^{2}-r^{2}\right)}{4\left(4 r^{2}-1\right)} \xi_{r-1}(x)$, the functions $\xi_{r}(x)$ satisfy the orthogonal relation

$$
\sum_{x=1}^{n} \xi_{i}(x) \xi_{j}(x)=0
$$

for $i \neq j$, any fixed $n$. The tabulated values $\xi_{r}^{\prime}$ are those of the smallest multiples of the $\xi_{r}$ that take integral values for every $x$. For $n \leqslant 8$, all individual values of $\xi_{r}{ }^{\prime}(x)$ are shown; for $n>8$, only those for which $x \geqslant \frac{1}{2} n$. The multiplier and the sum of squares over $x=1,2, \cdots, n$ are given for each $n, r$. With the aid of these tables the fitting of a polynomial up to degree 5 requires little more than the calculation of the sums of products of the observations with the corresponding tabular $\xi_{i}^{\prime}(x)$ values.

Table XXIV gives some formulae for the calculation of integrals from ordinates at equal intervals: (i) the coefficients of the Newton-Cotes formulae for 2, 4 and 6 panels with and without the use of an external ordinate: (ii) corresponding formulae expressed in terms of central differences up to the $10 \mathrm{th}$, for 2(2)10 panels: (iii) the first 16 coefficients in Gregory's formula.

Table VIII, reprinted from F. Yates, R. Statist. So., J. Suppl., v. 1, 1934, p. 228, is intended to provide an exact test of significance for the hypothesis that two observed fractions $a / b, c / d$ are estimates of the same true fraction; in other words, the test of independence in a $2 \times 2$ contingency table. The customary $\chi^{2}$ test, though improved by the correction for continuity, does not approximate the true significance levels very satisfactorily when $a, \cdots, d$ are small. The exact test necessitates a separate calculation for every set of values of $a, \cdots, d$. However, for all $2 \times 2$ contingency tables which have the same smallest expectation $m$, and the same ratio $p$ of the smallest expectation to the smallest marginal total, the exact significance levels of $\chi_{c}$ (corrected for continuity) lie between two limits which can be determined. These limits are tabulated (to $2 \mathrm{D}$ ) for $m=1(1) 6,8,12,24,48$, $96 ; p=0,0.25,0.5$, at the 0.025 and 0.005 significance levels of $\chi_{c}$, for each tail of the distribution.

Table XXII gives $\Delta^{r} 0^{s} / r$ ! for $s=2(1) 25 ; r=2(1) s$. This quantity occurs in the solution of a number of statistical problems. See $M T A C, \mathrm{p} .330$.

The basic plans for some common types of experimental arrangements may be constructed from Tables XV-XIX. Table XV gives a catalogue of the Latin squares from the $4 \times 4$ to the $6 \times 6$ (every standard square for the $4 \times 4$ and $5 \times 5$ and one square of each transformation set for the $6 \times 6$ ) and examples of Latin squares from the $7 \times 7$ to the $12 \times 12$. Table XVI contains complete sets of orthogonal Latin squares from the $3 \times 3$ to the $9 \times 9$, excluding the $6 \times 6$ for which no set exists. A number of balanced incomplete block arrangements are given in Table XVII. These are arrangements of $v$ different letters (representing experimental treatments) in groups of $k(k<v)$ such that any two letters appear together in a group the same number of times $(\lambda)$ : an example, for $v=4, k=2, \lambda=1$, is the set of groups $a b, a c, a d, b c, b d, c d$. Because of its symmetry, such an arrangement enables differences among the groups to be eliminated from the experimental errors of the treatment comparisons and is useful, for example, when the number of treatments exceeds the number that can be compared at any one time or place. Tables XVIII and XIX constitute indexes to these arrangements, by number of replications $(r)$ and number of units per group $(k)$ respectively, for all cases $(r \leqslant 10)$ in which the existence of an arrangement has not been disproved. For arrangements known by the authors to exist, references are given either to Table XVII or to simple methods of construction. 
In the layout of replicated experiments and in the process of taking samples, there is of ten occasion to use some randomizing device, such as a set of numbered beans. More convenient for many purposes is a set of random digits, of which 15,000 are given in Table XXXIII.

In addition, the volume contains Tables (XXV-XXXII) of some of the standard mathematical functions: $\log$ (to $5 \mathrm{D}$ ); $\operatorname{lns}$ (to $5 \mathrm{D}$ ); $n^{2}$ for $n=0(1) 999 ; n^{4}$, to $5 \mathrm{~S}$; reciprocals of $1.00(.01) 9.99$, to $6 \mathrm{D}$; factorials of $1(1) 300$ (to $6 \mathrm{~S}$ ) and $\log n !$, to $7 \mathrm{D}$; sines, to $5 \mathrm{D}$, and tangents, mostly to $5 \mathrm{D}$. The table of square roots is very convenient, with $\sqrt{n}$ and $\sqrt{10 n}$ placed one above the other. The tabulation of $\left(90^{\circ}-\theta\right) \tan \theta$ instead of $\tan \theta$ for $\theta$ above $60^{\circ}$ may also be noted.

Notes on the use of the tables occupy the first 22 pages. For some Tables, e.g. XX and XXIV, the notes might profitably be amplified.

The tables are admirably printed on large pages with open-flat binding and are comfortable to the eye. As the early issue of a second edition has indicated, this volume is a most useful addition to the statistical library of the research worker. For the few errors in the tables, see MTE 47.

In the second edition four tables have been added. Tables $V_{1}$ and $V_{2}$ give a test of significance of the difference between the means of two samples, for the case where the variances in the two populations are not assumed to be equal. The development of a test of this kind has encountered interesting problems and raised some controversy. On the classical theory of probability, the simplest approach might be to work the frequency distribution of $d=\left(x_{1}-x_{2}\right) / \sqrt{s_{1}^{2}+s_{2}^{2}}$, where $s_{1}{ }^{2}, s_{2}{ }^{2}$ are the sample estimates of the variances of $\bar{x}_{1}, \bar{x}_{2}$ respectively, for populations whose variances $\sigma_{1}^{2}, \sigma_{2}^{2}$ have fixed though unknown values. It has been known for some time, however, that this distribution depends on the unknown ratio $\sigma_{1}{ }^{2} / \sigma_{2}{ }^{2}$ and consequently cannot supply an exact test of significance. Another method, H. SchefFE, Ann. Math. Statist. v. 14,1943, p. 35-44, is to select a different test criterion whose distribution is that of "Student's" $t$. This technique involves the use of an estimate of the variance of $\left(\bar{x}_{1}-\bar{x}_{2}\right)$ which is generally less precise than $\left(s_{1}{ }^{2}+s_{2}{ }^{2}\right)$. Fisher's solution, proposed first by W. V. BEHRENS, Landwirtschaftliche Jahrbücher v. 68, 1929 , p. $807-837$, is to regard the ratio $\sigma_{1}{ }^{2} / \sigma_{2}{ }^{2}$ as following its fiducial distribution while $s_{1}{ }^{2} / s_{2}{ }^{2}$ remains fixed. In effect, the "nuisance" parameter $\sigma_{1}{ }^{2} / \sigma_{2}{ }^{2}$ is integrated out of the classical solution.

For practical purposes the important question is whether the mathematical model describes the conditions under which the test is applied. If, for example, the test were used repeatedly for the comparison of experimental results on a certain crop, it would be taken for granted that $\sigma_{1}^{2} / \sigma_{2}^{2}$ varies from one application to another, though probably the complete fiducial distribution would not be followed since there is a positive lower limit to the error variances in field experiments. In these circumstances, over a long series of applications the significance levels might be reasonably correct. In Table $V_{1}$, due to $\mathrm{P}$. V. SuKHATME, $n_{1}, n_{2}$-numbers of degrees of freedom in $s_{1}{ }^{2}, s_{2}{ }^{2}$ respectively $=6,8,12,24, \infty$; $\theta=\tan ^{-1}\left(s_{1} / s_{2}\right)=0^{\circ}\left(15^{\circ}\right) 90^{\circ} ; P=$ significance level $=.05, .01 ; d$ to $3 \mathrm{D}$. Table $V_{2}$ applies to the case where $n_{1}$ is infinite; $n_{2}=10,12,15,20,30,60, \infty ; \theta=0^{\circ}\left(10^{\circ}\right) 90^{\circ}$; $P=.10, .05, .02, .01, .005, .002 ; d$ to $3 D$. The authors state that from comparison with Table $V_{2}$, the values in Table $V_{1}$ may be too high by about .001 .

When a series of $N$ independent trials of an event shows $a$ successes, Table VIII (W. L. Stevens) provides upper and lower fiducial limits to the expected number of successes for significance levels $P=0.1,0.025,0.005$. The tabulated values are $N p$ (mostly $2 \mathrm{D}$ ) where for an upper level

$$
P=\sum_{r=0}^{a} \frac{N !}{r !(N-r) !} p^{r} q^{N-}
$$

$a=0(1) 14, a / N=.5(-.1) 0$, except for $a<4$ where more values of $a / N$ are given. For $a>15, p=.5(-.1) 0$, corrections are given to the estimates derived from the normal approximation to the binomial. The authors suggest (p. 25) that these corrections are not 
quite exact for $a>15$. Fiducial limits of the Poisson distribution are obtained from the value for $a / N=0$.

In the dilution method for estimating the number of certain organisms in a solution, a series of $s$ concentrations is made, each $a$ times as dilute as the previous one. For each concentration $n$ tubes are prepared and the presence or absence of the organism is noted for every tube. If $\lambda$ is the true number of organisms per tube at the highest concentration, the number in the $r^{\text {th }}$ dilution is $\lambda / a^{r}$. The theory of the method is that for this level the numbers of tubes having $0,1,2, \cdots$ organisms are distributed in a Poisson series with mean $\lambda / a^{r}$. Hence the probability of a fertile tube is $\left(1-e^{-\lambda / a^{r}}\right)$ and the expected number of fertile tubes is

$$
X=n s-n\left(e^{-\lambda}+e^{-\lambda / a}+\cdots+e^{-\lambda / a \sigma-1}\right) .
$$

If $X$ is taken as the observed number of fertile tubes, this equation supplies an estimate of $\lambda$ which, though not fully efficient, discards only about 12 per cent of the information. Solutions of the equation are provided in Table $\operatorname{VIII}_{2}$ for $a=2,4,10$. With two-fold dilution, $s=4(1) 10$, plus a single set of solutions for $s>10 ; x=X / n=.4(.2) 2(.5)$ $\overline{s-2}(.2) \overline{s-.4}$. For $a=4, s=4,5,>6$ and for $a=10, s>3$. The function tabulated $(3 \mathrm{D})$ is a quantity $k$ such that

$$
\log \lambda=x \log a-k .
$$

Table XVII (Balanced incomplete blocks) has been revised so as to include four solutions discovered since the appearance of the first edition.

Explanatory notes have been added for the new tables and some revisions made in the previous notes.

Iowa State College of Agriculture

W. G. CochraN

and Mechanic Arts

165[L].-David Moskovitz, "The numerical solution of Laplace's and Poisson's equations," Quart. Appl. Math., v. 2, 1944, p. 148-163. $17.8 \times 25.3 \mathrm{~cm}$.

Using the approximate method of difference equations, based on a lattice of meshwidth $h$, the author has solved the two-dimensional Laplace and Poisson equations for a rectangle: $0 \leqq x \leqq n h, 0 \leqq y \leqq m h$; the values of the solution $u$ on the boundary are assumed given. General formulae are given in the text and tables are provided from which the solutions can be quickly computed in the cases $m=2,3$, or $4, n$ being an arbitrary integer. To use the tables, in the case of the Laplace equation, one substitutes the given boundary values in the left hand column, multiplies each entry there by the corresponding number in the proper $n$ column, and then adds the results. In this way the second table yields $u_{1}(2)=u(2 h, h)$ in the case $m=2$. Using this new value, one now has a new boundary value problem for a smaller rectangle, for which the process can be repeated. In this way the function is computed at every other point. Finally, by the fundamental interpolation formula, one finds the values at the points skipped over. Similar procedures are used for the other cases. For the Poisson equation the values of the right hand member are weighted by multipliers and added in similarly. In the text there are instructions for modification of the procedure for a rectangle: $0 \leqq x \leqq n h, 0 \leqq y \leqq(m+r) h$, where $n$ and $m$ are integers, but $0<r<1$. The entries of the tables are rounded off to $4 \mathrm{D}$ from calculations carried out to a larger number of decimal places.

The chief disadvantage of the results is that in the tables $m$ is restricted to the values 2,3 and 4 . However, as the author remarks, one can use a $4 \times n$ lattice as a first approximation, after which relaxation methods can be applied to a finer mesh.

Brown University

WILFRED KAPLAN 
166[L].-Mitiyasu TAKagi, "On a statistical theory of ferromagnetic crystals. Part I. Magnetization and magnetostriction," Tôhoku Imp. Univ., Science Reports, s. 1, v. 28, 1939, p. 20-84. $18.7 \times 26.5 \mathrm{~cm}$.

The appendix to this paper, p. 73-84, contains an important table of the Bessel function $I_{n}(x)$, together with a short description of the interesting method used for computation. The tables include $I_{n}(x)$ to $12 \mathrm{~S}^{1}$ for $x=1(1) 6, n=0(1) 11$, taken from GRAY \& MATHEWs ; these values came in the first place from the BAASMTC report for 1889 . The remaining tables are original and are to $10 \mathrm{~S}$ for $n=0(1) N$, with a 'tail' of $9 \mathrm{~S}$ to $5 \mathrm{~S}$ values for $n$ up to $N^{\prime}$, where $N$ and $N^{\prime}$ are as follows:

\begin{tabular}{l|rrrrrrrrrrrrr}
$x$ & 7 & 8 & 9 & 10 & 20 & 30 & 40 & 50 & 60 & 70 & 80 & 90 & 100 \\
$N$ & 23 & 23 & 25 & 25 & 29 & 34 & 39 & 42 & 44 & 47 & 49 & 53 & 54 \\
$N^{\prime}$ & 25 & 26 & 27 & 28 & 34 & 39 & 43 & 47 & 50 & 53 & 57 & 61 & 64
\end{tabular}

Except for a few isolated entries, these tables cover a new range in published values; the writer, however, has access to Ms. tables ${ }^{3}$ calculated during preparation of a second volume of tables of Bessel functions by the BAASMTC. Values common to both tables (that is all Takagi's values for $x=7(1) 10$ and for $x=20$ up to $n=30$ ) have been compared; the comparison shows his values to be correct except that, as he expected, the last figure is not reliable. In the 10-figure part of the table this last figure is in error by maximum amounts of 1 or 2 units when the leading digit is small, and up to 6 or 7 units when the leading digit is an 8 or 9 , that is, the error tends to be a proportionate error of maximum amount about $7 \times 10^{-10}$. In the tails the errors are greater, up to 2 digits being affected.

It is of interest to refer back to MTE 43, p. 200, in which J. W. WRENCH, Jr. discusses two discrepant values of $I_{0}(30)$, giving his own result

Takagi gives

$$
I_{0}(30)=781672297823 \cdot 97748972
$$

$$
=781672297900
$$

whilst the BAASMTC value (obtained by W. G. BICKLEY), retaining guard figures, is $781672297823 \cdot 9774900$.

\section{J. C. P. Miller}

1 The value of $I_{11}(3)$ has $11 S$ only in each table.

2 A. GRAY and G. B. Mathews, A Treatise on Bessel Functions, London, Macmillan, 1895, p. 285. Second edition, prepared by A. Gray and T. M. MAcRoBerT, 1922, p. 309.

${ }^{3}$ Described in $M T A C$, p. 283.

167[M].-1. David Bierens De HaAN (1822-1895), Nouvelles Tables d'Intégrales Définies, Edition of 1867 corrected with an English translation of the Introduction by J. F. RirT. Off-set print: New York, G. E. Stechert \& Co., 1939. xviii, 716 p. $21.5 \times 27 \mathrm{~cm} . \$ 15.00$.

2. Christian Fredrik Lindman (1816-1901), Examen des Nouvelles Tables d'Intégrales Définies de M. Bierens de Haan, Amsterdam, [sic] 1867. (K. Svenska Vetenskaps Akad., Handlingar, v. 24, no. 5, Stockholm, 1891.) Off-set print: New York, G. E. Stechert \& Co., 1944, 231 p. $21.5 \times 26.8 \mathrm{~cm} . \$ 7.50$.

Apart from having achieved fame as the outstanding mathematical bibliographer and historian of the Netherlands, Bierens de Haan put all mathematicians in his debt as the result of enormous labor in assembling and tabulating material regarding definite integrals and their values. His first great collection of about 7300 formulae was published in 1858 and filled v. 4 of the Verhandelingen, of the Royal Academy of Sciences of Amsterdam. But Bierens de Haan continued active research in the field and by 1862 had filled the 702 p. of v. 8 of the Verhandelingen, with an Exposé de la Théorie des Propriétés, des Formules 
de Transformation, et des Méthodes d'Évaluation des Intégrales Définies. A few years later, having accumulated considerably more material he finally published at Leiden in 1867, with the aid of a royal subsidy, the volume of Nouvelles Tables, containing 8359 formulae, of which only about 4200 were in the volume of 1858 . This earlier collection was still valuable, however, not only because of some 3000 integrals not used in the new edition, but also on account of numerous literature references omitted in the later condensation. The thousands of definite integrals and their values were almost wholly arranged in the new work in 486 "tables," mainly grouped in accordance with the functions integrated, or with the limits of integration. In the original work there were "Additions," p. 699-727 and "Corrections," p. 729-733.

Up to 1939 there was always a chance each year to pick up copies of this 1867 edition for $\$ 15.00$ a copy, or less, although other copies were frequently priced much higher. With war's disruption of international second-hand book markets came the American edition. Since this edition has only $716 \mathrm{p}$. in the main body of the work, while the original edition had (as we have seen) 733 p., anyone who had not seen both editions might incorrectly infer that there was less material in the American edition. But this is not the case. All that is on p. 699-727 (with many blank pages) of the 1867 edition, is on p. 699-716 of the American edition; nearly 300 errors listed in 1867 on five pages were abolished in 1939, because all the errors in question were then corrected in the body of the text. Unfortunately these were the only errors corrected in the preparation of this edition although many others had been pointed out nearly 50 years previously.

Already in 1884 Lindman, a gymnasium mathematics teacher, had published in the Bihang of the Swedish Academy of Sciences, v. 10, no. 3, "Observations sur les tables d'intégrales définies de M. Bierens de Haan" (Amsterdam, 1858), $268 \mathrm{p}$. Then seven years later, in the Handlingar, appeared his listing of the errors in the 1867 tables. This is the work which has now been reproduced and made available for other libraries than the few which happened to possess the volume of the Swedish serial in question.

Even if it is assumed that most of the errors of the 1867 collection of tables are eliminated by the Lindman publication, the work of nearly three quarters of a century ago is wholly inadequate for modern research needs. It has been estimated that an up-to-date comprehensive table of the definite integrals now available would probably fill three or four large volumes each containing 600 to 700 pages. It was mainly with a view to providing for publication of such a work by HARRY BATEMAN that a revolving fund was secured for our Committee. All scientific workers must applaud such a magnificent undertaking, and hope for its completion in the not too distant future.

R. C. A.

168[M].-H. M. Terrill \& Lucile Sweeny, "An extension of Dawson's table of the integral of $e^{x^{2}}$," Franklin Institute, J., v. 237, 1944, p. 495-497.

This contribution from the Biochemical Research Foundation at Newark, Delaware, is an extension of a table of $I=\int_{0}^{x} e^{x^{2}} d x$, for the range $x=[.01(.01) 1.99 ; 6 \mathrm{D}]$ and $x=[2 ; 5 \mathrm{D}]$, by H. G. Dawson in London Math. So., Proc., v. 29, 1898, p. 521-522. The extension is for the range $x=[2(.01) 4 ; 7-9 \mathrm{~S}]$.

In preparing this extension the method followed consisted in first computing, from $x=1.9$, by direct methods, the values of the function for 22 key arguments, differing by .1 , and then filling in the values for the intermediate arguments by Gregory's formula. For the direct computation two series were employed checking against each other, the first being that of Dawson, and the second the uniformly convergent series

$$
I=x+x^{3} / 3+x^{5} /(5.2 !)+x^{7} /(7.3 !)+\cdots .
$$

"Since this series is the same as the series for the probability integral, save for the signs of 
alternate terms, only half the terms are needed. The terms of odd rank are computed, and from twice the sum of these the appropriate value of the probability integral is subtracted."

The computation was started at $x=1.9$ to enable a check to be made with some of Dawson's results; for $x=1.92$, the authors found that $I=12.70733$, instead of Dawson's value 12.703175. For other Dawson errors see MTE 46. The values of the key arguments were computed $\geqslant 10 \mathrm{~S}$, and to $7 \mathrm{D}$ for the intermediate ones, except at the extreme end of the table.

The integral $I$ appears in problems of mathematical physics, hydrodynamics, etc. H.B. has supplied the following references (the authors of the table under review make no acknowledgment that information concerning references 4-5 below was supplied to them):

1. K. Terazawa, R. So. London, Proc., v. 92A, 1916, p. $68 ; g(x)=e^{-x^{2}} \int_{0}^{x} e^{t^{2}} d t$, as well as its first and second derivatives, are tabulated, $x=[0(.1) 1(1) 3,5,10 ; 3-5 \mathrm{D}], x^{2}=2,3,5,7$.

2. K. Terazawa, Tôhoku Univ., Sci. Reports, v. 6, 1917, p. 172-173; tables of $g(x)$ and first four derivatives $x=[0(.1) 1,4,5,10 ; 3-5 \mathrm{D}], x^{2} / 2=.6(.2) 6$. These functions arise in consideration of the oscillation of a deep-sea surface caused by a local disturbance.

3. E. E. Watson, Phil. Mag., s. 7, v. 3, 1927, p. 850 , graph of $F(R)=\int_{1}^{R} d x(\ln x)^{3}$ $=2 \int_{0}^{(\ln R)^{2}} e^{t^{2}} d t, 0<R<7$; in discussing the dispersion of an electron beam.

4. N. KaPzov \& S. Gwosdower, Z.f. Physik, v. 45, 1927, p. 133, the function $x \cdot e^{-x^{2}} \int_{0}^{x} e^{t^{2}} d t$ is tabulated for $x=[.1, .5, .8(.2) 1.2(.05) 2.2 ; 5 \mathrm{D}] ; \Delta, 1.5-2.2$. The use for such a table arises in discussion of oscillations in electron tubes.

5. S. Sakomoto, Sächs. Akad. d. Wissen., Berichte ü. d. Verh., math.-phys. Kl., v. 80, 1928, p. 217-223, who gives tables of $g(x)$ and $g(x) / x$ for $x=[0(.01) 10 ; 4 D]$; they were needed in a problem of heat conduction under certain boundary conditions.

6. W. L. Miller \& A. R. Gordon, J. Phys. Chem., v. 35, 1931, p. 2878-2882, g(x) for $x=0(.01) 4(.05) 7.5(.1) 10(.2) 12 ; 0-1.99 ; 6 \mathrm{D}, 2-4.95 ; 8 \mathrm{D}, 5-12 ; 9 \mathrm{D}$. The computation for $x=0-2$ was based on the uncorrected Dawson table.

In the Library of Brown University the authors have deposited a Ms. giving the values of $I$ as follows:

$$
x=[0(.01) .1(.1) 1.8 ; 10 \mathrm{D}],[1.9(.1) 4 ; 5-9 \mathrm{D}] .
$$

R. C. A.

\section{MATHEMATICAL TABLES-ERRATA}

References have been made to errata in no. 7 of $M T A C$, part II, Bibliography, under Airey 19, Airy 4, 5, Bessel, Bourget, BAASMTC 1, Carrington, Colwell \& Hardy, Dale, Davis \& Kirkham, Dinnik 8, 9, 10, 11, 14, Doodson, Gray \& Mathews, Hayashi, Jahnke \& Emde (also under Bisacre), Kalähne, Karas, Lehmer, Lommel 2, 3, Maclean, Meissel 1, 5, NYMTP 3, Nicholson 1, Rayleigh 8, A. Russell (under Maclean), Schleicher, Schulze, B. A. Smith 1, Steiner, Tölke, Watson, A. G. Webster, Willson \& Peirce. See also RMT 163 (Davis), 164 (Fisher \& YATES), 166 (TAKAGI), 167 (BIERENS DE HAAN), 168 (DAWSON); UMT 24 (Potin); MAC 11 (AdAMs); N 21 (DEgEN, Wrinch).

44. BAASMTC, Mathematical Tables, v. 1, London, 1931.

P. $5, \cos 26.1$, for $.56756 \cdots$, read $.56755 \cdots$

P. 7 , sin 47.6 , for $.46832 \cdots$, read $.45832 \ldots$

It will be noted that in both cases a 5 has been converted to a 6 .

L. J. C. 